Fixed Point Theory, 20(2019), No. 1, 271-288

DOI: $10.24193 /$ fpt-ro.2019.1.18

http://www.math.ubbcluj.ro/ nodeacj/sfptcj.html

\title{
A NOTE ON A STRUCTURE THEOREM FOR PREHOMOGENEOUS VECTOR SPACES
}

\author{
MASAYA OUCHI \\ Faculty of Education and Human Studies Course, Akita University \\ Akita, 010-8502, Japan \\ E-mail: m-ouchi@math.akita-u.ac.jp
}

\begin{abstract}
In this note, we give a structure theorem for all prehomogeneous vector spaces defined over the complex number field $\mathbb{C}$. Also it means a necessary and sufficient condition for a triplet $(G, \rho, V)$ defined over $\mathbb{C}$ to be a prehomogeneous vector space. For this purpose, we give a general structural correspondence between isotropy subgroups and fixed point sets when a group acts on a non-empty set.

Key Words and Phrases: Prehomogeneous vector space, representation theory of groups, fixed point.
\end{abstract}

2010 Mathematics Subject Classification: 11S90, 20Cxx.

Acknowledgments. The author would like to express the most sincere gratitude to Professor Emeritus Tatsuo Kimura for cordial supervision and essential advices. And, many important advices given by Dr. Yoshiteru Kurosawa are indispensable to this paper. The author dedicates this paper to them with the deepest admiration. Finally, the author is most grateful to the referees for valuable comments and accurate corrections to the description.

\section{REFERENCES}

[1] A. Borel, Linear Algebraic Groups, Second Edition, Graduate Texts in Mathematics, 126(1991).

[2] A. Gyoja, A theorem of Chevalley type for prehomogeneous vector spaces, J. Math. Soc. Japan, 48(1996), 161-167.

[3] S. Kasai, A classification of reductive prehomogeneous vector spaces with two irreducible components, I, Japan. J. Math., 14(1988), 385-418.

[4] T. Kimura, A classification of prehomogeneous vector spaces of simple algebraic groups with scalar multiplications, J. Algebra, 83(1983), no. 1, 72-100.

[5] T. Kimura, Introduction to prehomogeneous vector spaces, Translations of Math. Monographs 215, Amer. Math. Soc., Providence, RI, 2003.

[6] T. Kimura, S. Kasai, M. Inuzuka, O. Yasukura, A classification of 2-simple prehomogeneous vector spaces of type I, J. Algebra, 114(1988), 369-400.

[7] T. Kimura, S. Kasai, M. Taguchi, M. Inuzuka, Some P.V.-equivalences and a classification of 2-simple prehomogeneous vector spaces of type II, Trans. Amer. Math. Soc., 308(1988), 433-494.

[8] T. Kimura, K. Ueda, T. Yoshigaki, A classification of 3-simple prehomogeneous vector spaces of nontrivial type, Japan. J. Math. (N.S.), 22(1996), no. 1, 159-198. 
[9] Y. Kurosawa, On a classification of 3-simple prehomogeneous vector spaces with two irreducible components, Tsukuba J. Math., 36(2012), no. 1, 135-172.

[10] M. Ouchi, A classification of reductive prehomogeneous vector spaces with trivial representation free, Tsukuba J. Math., 33(2009), no. 1, 177-188.

[11] M. Sato, T. Kimura, A classification of irreducible prehomogeneous vector spaces and their relative invariants, Nagoya Math. J., 65(1977), 1-155.

Received: October 6, 2016; Accepted: March 20, 2017. 
